\title{
Study of the Influence of Nanoparticles on the Molecular Model of an Ideal Fluid
}

\author{
Mahammadali Ahmad oglu Ramazanov1, Mammad Samad oglu Aslanov² \\ ${ }^{1}$ Baku State University, Baku, Azerbaijan \\ ${ }^{2}$ Azerbayjan Technical University, Baku, Azerbaijan \\ Email: mamed r50@mail.ru,nanomaterials@bsu.az
}

Received 28 July 2015; accepted 24 May 2016; published 27 May 2016

Copyright (C) 2016 by authors and Scientific Research Publishing Inc.

This work is licensed under the Creative Commons Attribution International License (CC BY). http://creativecommons.org/licenses/by/4.0/

(c) (i) Open Access

\begin{abstract}
In the article a molecular model of oil with nanoparticles on the basis of the model of ideal fluid is considered. It is assumed that the molecular model of the oil can be represented as a homogenous distribution of identical molecules in space. It is assumed that the central interaction between the oil molecules and nanoparticles, results in a change of the model parameters. It is shown that for an ideal fluid the effect of nanoparticles is reduced to a change of the coefficient at the pressure.
\end{abstract}

\section{Keywords}

Ideal Fluid, Molecular Model of Oil, Nanoparticles, Coefficient of Elasticity

\section{Introduction}

At the present time the development of effective technologies, based on nanotechnology in the fields of oil, automotive, shipbuilding, construction, aviation industries and etc. is highly evaluated. For example, the application of nanotechnology in the oil industry is associated with an increase of the oil recovery factor of highviscosity oil fields and in this direction was carried out numerous studies [1]-[7].

Developed countries spend significant expenditures on the development of oil science that allows creating cost-effective technologies for the extraction of hard recoverable oil reserves.

According to the material of the London Forum on oil recovery, applications of already developed advanced technologies allow raising the average recovery factor up to $50 \%$ by 2020 , which in turn increase oil recovery of the proven world's oil reserves on 1.4 times [3].

It is known that the oil is a complex mixture of hydrocarbons and carbon compounds. Not separated into individual components, it can be accepted as an ideal fluid in a molecular level.

So, study of the model of an ideal fluid containing nanoparticles is of scientific interest and is very relevant in 
the nanotechnology field.

\section{Formulation of the Problem}

It is known that the liquid has a continuum and when it is at rest or moves as a rigid body, only the normal stress is observed and at the same time there is no shear stress.

In real fluids shear stresses are not equal to zero, but too small and incomparable to the normal stresses. In such cases, the liquid is taken as the ideal fluid [3].

In contrast to the continuum hypothesis when considered liquid system is described on the molecular scale, it is necessary to model the system by molecular dynamics. The essence of this method is as follows: an ensemble of particles formed from atoms and molecules that is individually accepted as material points. It is assumed that the particles containing atoms and molecules interact to each other and can be subjected to external interaction. Interatomic or intermolecular interactions are described by means of van der Waals forces, which are mathematically expressed by Lennard-Jones potential [3].

In the nature a real fluid characterised by the physical properties of an incompressible fluid that has constant density and the friction force between the particles. Due to difficulty of describing of hydrodynamic processes in such fluids and in order to simplify the solving of tasks relating them the concept of an ideal fluid is introduced. It is assumed that the viscosity of an ideal fluid is equal zero, i.e., there is no friction between the particles. Despite the fact of ideal fluid does not exist, these assumptions facilitates the study of the mechanical properties of real liquids and solutions of some problems.

Required to identify the impact of nanoparticles on the molecular model of an ideal fluid.

\section{The Solution of the Problem}

Assume that the ideal liquid is the body, wherein the tension in all directions is equal.

Based on this assumption the molecular model of oil can be represented as uniform distribution of identical molecules in space. There is a central interaction between these molecules. We accept the strength of the interaction $F(a)$ elastic and low, i.e.

$$
F(a) \approx F\left(a_{0}\right)+F^{\prime}\left(a_{0}\right) \cdot \Delta a \approx c \cdot \Delta a,
$$

where $a_{0}$-the initial distance, $a$-the distance between the interacting molecules, $\Delta a=a-a_{0}, c$-the coefficient of elasticity (Figure 1).

For the construction of the defining equation draw the plane. We distinguish molecules with the smallest distance to the plane. Draw a line perpendicular to the plane. Their numbers $N,(N=4)$. The total force is equal to $(N-1) a \sigma$, where $\sigma$-stress. On the other hand

$$
-(N-1) a_{0} \sigma=(N-k) F(b)+F(d) \cdot \frac{b_{0}}{a_{0}} \cdot(N-k) \cdot 2
$$

where $d^{2}=b^{2}+a^{2} ; k$ - the number of planes that do not have contact (Figure 2).

From (1) we obtain

$$
-\sigma=\frac{N-k}{N-1} \cdot \frac{1}{a_{0}} \cdot F(n)+\frac{N-k}{N-1} \cdot \frac{b_{0}}{a_{0}^{2}} \cdot 2 F(d) \approx \frac{1}{a_{0}} \cdot F(b)+\frac{b_{0}}{a_{0}^{2}} \cdot 2 F(d) .
$$

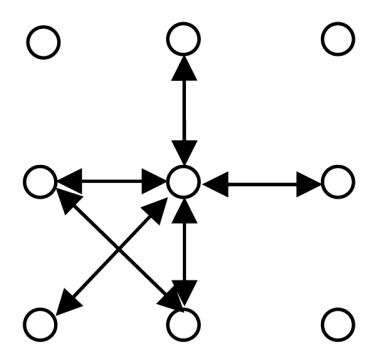

Figure 1. Scheme of the interaction between molecules. 
Since $\sigma$ is not dependent on the angle of inclination of the plane, then $F(d) \approx 0$

$$
-\sigma=\frac{1}{a_{0}} \cdot F(b)=\frac{1}{a_{0}} \cdot c \cdot \Delta b=\frac{b_{0}}{a_{0}} \cdot c \cdot \varepsilon, \quad \varepsilon=\frac{\Delta b}{b_{0}} .
$$

From (2) it follows that the voltage in an ideal fluid is, the compressive stress and no slip, i.e.

$$
\sigma_{i j}=-p \delta_{i j}, \quad p=\frac{b_{0}}{a_{0}} \cdot c \cdot \varepsilon, \quad \delta_{i j}=\left\{\begin{array}{l}
1, \text { nрu } i=j \\
0, \text { npu } i \neq j
\end{array}\right.
$$

where $\delta_{i j}$-the Kronecker symbol (Figure 3).

If $b_{0}=a_{0}$, then we get $p=c \cdot \varepsilon$.

The physical meaning of $\varepsilon$-the relative change in volume, i.e. $p$-depends on $\varepsilon$ or $p$ depends of the density (at constant mass the volume change leads to a change in density).

Addition of nanoparticles leads to a change of the molecular model of oil. Then, the liquid keeps staying ideal, or will become non-ideal. In case the liquid keep staying ideal, then molecular model takes the form (see Figure 4).

In the I and II models there are sliding (shear) i.e. these are non-ideal fluids. In the III model the interaction is central.

Drawing the plane through the liquid medium containing nanoparticles, we get the following model (Figure 5).

I row is not considered as the closest row is the row of nanoparticles. Then

$$
-\sigma=\frac{2}{a_{0}} F\left(\frac{b}{2}\right) \approx \frac{2}{a_{0}} c_{n} \Delta \frac{b}{2}=\frac{2}{a_{0}} \cdot \frac{b_{0}}{2} \cdot c_{n} \varepsilon_{n}=\frac{b_{0}}{a_{0}} c_{n} \varepsilon_{n}, \quad \varepsilon_{n}=\frac{\Delta \frac{b}{2}}{\frac{b_{0}}{2}}=\varepsilon,
$$

where index " $n$ " means, that this value refers to nanoparticles.
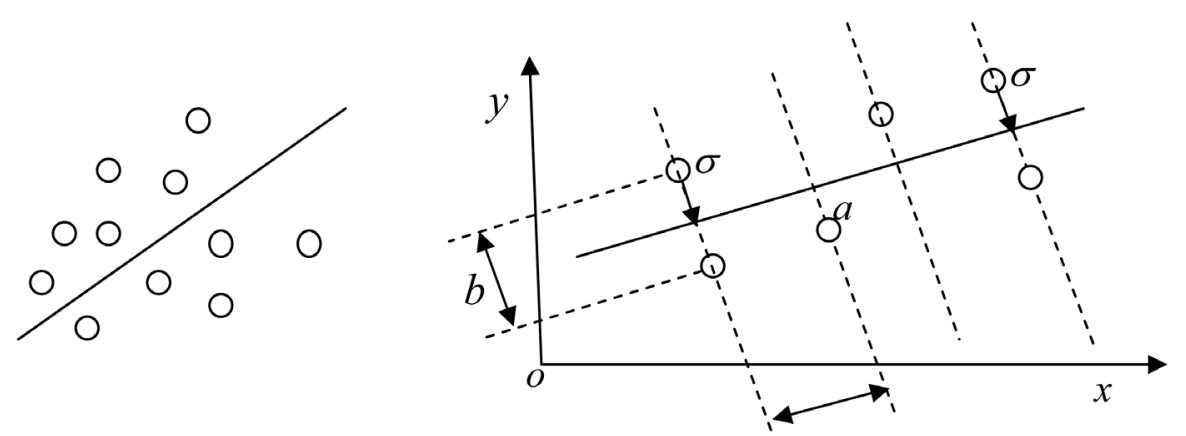

Figure 2. The scheme of arrangement of the molecules relatively to the plane separating the liquid medium.

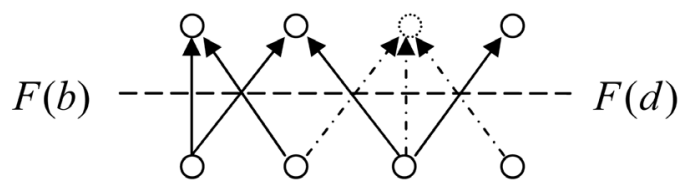

Figure 3. The scheme of interactions of atoms in the presence of vacancies.
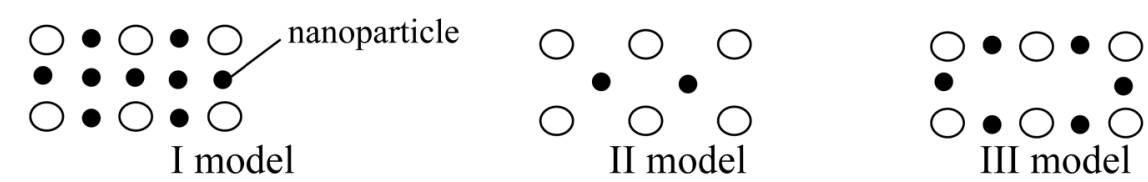

Figure 4. Molecular models of the oil with the addition of nanoparticles. 


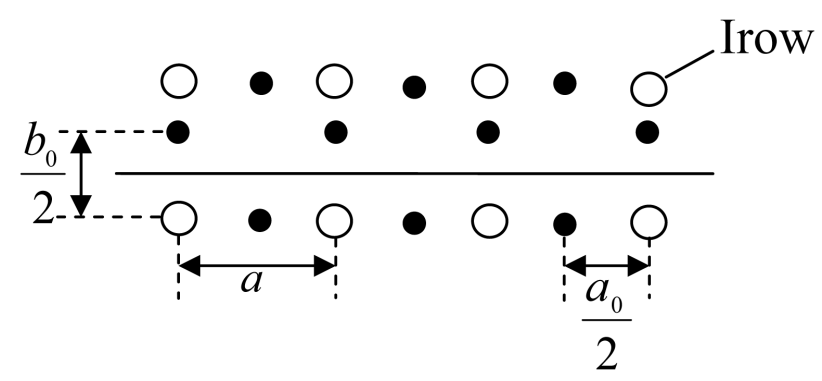

Figure 5. The scheme of arrangement of the nanoparticles relatively to the plane separating the liquid medium.

Finally, we find that

$$
-\sigma=\frac{b_{0}}{a_{0}} c_{n} \varepsilon=c_{n} \varepsilon=\frac{c_{n}}{c} p .
$$

Thus, for an ideal fluid the effect of nanoparticles is reduced to a change of the coefficient at pressure.

Note that non-ideal fluid is a body in which the voltage is a superposition of the voltage of an ideal fluid and shifting, i.e., this ideal fluid is taken with consideration of shear deformation. Hence we can say that non-ideal fluid is the body, which changes its shape, but does not change the volume $(p=0)$.

Molecular model of non-ideal fluid is the same that for ideal fluid but the molecules can participate simultaneously in two (or more) of motion, i.e. there is possible shift.

\section{Conclusion}

In the article a molecular model of oil with nanoparticles on the basis of the model of ideal fluid is described. It is assumed that the molecular model of the oil can be represented as a homogenous distribution of identical molecules in space. It is assumed that the central interaction between the oil molecules and nanoparticles, results in a change of the model parameters. It is shown that for an ideal fluid the effect of nanoparticles is reduced to a change of the coefficient at the pressure.

\section{References}

[1] Khavkin, A.J. (2008) Nanotechnology in Oil and Gaza. The Campaign Sputnik, 149 p.

[2] Sergeev, G.B. (2007) Nanochemistry: Textbook. 2nd Edition, KDU, 336 p.

[3] Abdullayev, S. and Nagiyev, F. (2011) Nanogidromehanika. Research Center of High Technologies. Baku, 158 p.

[4] Mirzadzhanzade, A.Kh., Maharramov, A.M., Yusifzade, A.Kh., Shabanov, A.L., Nagiyev, F.B., Mammadzade, R.B. and Ramazanov, M.A. (2005) The Study of the Influence of Nanoparticles of Iron and Aluminum in the Process of Increasing the Intensity of Gas Evolution and Pressure to Use in Oil Extraction. News Baku State University, No. 1, 5-13.

[5] Alizadeh, A.N. (2010) Tension of a Plate with Nanomaterial Coating. Reports of National Academy of Sciences of Azerbaijan, 66, 34-43.

[6] Ramazanov, M.A. and Aslanov, M.S. (2011) Maxwell Model Based Nanoparticles. The International Symposium "Fullerenes and Nanostructures in Condensed Matter", Collected Articles, National Academy of Sciences of Belarus, Minsk, Center BSU, 14-17 June 2011, 229-234.

[7] Ramazanov, M.A. and Aslanov, M.S. (2013) The Change of Physical-Mechanical Properties of Viscoelastic Liquids in Consideration of Nanoparticles. Applied Mathematics, 4, 1367-1370. http://dx.doi.org/10.4236/am.2013.410184 